\title{
RETORNANDO A CHAITÉN: DIAGNÓSTICO PARTICIPATIVO DE UNA COMUNIDAD EDUCATIVA DESPLAZADA POR UN DESASTRE SOCIONATURAL
}

\author{
ADRIANA E. ESPINOZA S. ${ }^{a}$, CLAUDIA E. ESPINOZA Z. ${ }^{b} \&$ ANITA A. FUENTES P. ${ }^{c}$
}

\begin{abstract}
RESUMEN
Chile es un país que se caracteriza por los desastres socionaturales que periódicamente afectan el territorio. Entre ellos destacan terremotos, tsunamis, aluviones, incendios forestales y erupciones volcánicas. En el año 2008 se produce la erupción del volcán Chaitén que obligó a evacuar a más de 1.500 familias, quienes debieron emprender un largo proceso de desplazamiento forzado y en muchos casos un retorno posterior. Este complejo proceso incluye también a instituciones como las escuelas y sus respectivas comunidades educativas.

En este artículo se presenta un diagnóstico participativo realizado en el contexto de un proceso de intervención/investigación por un equipo de intervención de la Universidad de Chile, con los miembros de la comunidad educativa Juan José Latorre, primera escuela habilitada en Chaitén luego del retorno en el año 2010. Los resultados de este diagnóstico participativo permiten obtener una visión panorámica del proceso vivido por esta comunidad, los que dan cuenta del contexto educativo antes de la erupción, durante el proceso de desplazamiento y el posterior retorno. Visibilizando la importancia de la escuela como foco de atracción para el retorno de muchas familias. Se presentan las estrategias metodológicas de abordaje utilizadas y las temáticas que surgen de este proceso investigativo, destacando la importancia de indagar sobre los efectos en la salud mental producidos por estos eventos en la comunidad educativa y su impacto en la relaciones interpersonales así como en las nuevas demandas educativas que surgen en contexto de retorno. Finalmente, se analiza la pertinencia de esta metodología de intervención/investigación en los procesos de reconstrucción psicosocial posteriores a desastres socionaturales.
\end{abstract}

PALABRAS CLAVE: Desplazamiento, desastres socionaturales, erupción volcánica, Chaitén, Chile.

a Ph.D., Académica del Departamento de Psicología de la Universidad de Chile, Investigadora Asociada Centro de Investigación en Vulnerabilidades y Desastres Socionaturales (CIVDES), Núcleo Científico Milenio NS100022, Ignacio Carrera Pinto 1045 , Nuñoa, Chile, aespinoza05@vtr.net

b Psicóloga y Magíster en Psicología Educacional Universidad de Chile. Tesista Centro de Investigación en Desastres Socionaturales (CIVDES), Núcleo Científico Milenio NS100022, claudiaez@u.uchile.cl

c Psicóloga y Universidad de Chile. Arteterapeuta. Postítulo de especialización en Terapias de Arte. Mención Arteterapia. Universidad de Chile. Coordinadora de proyecto Valentín Letelier: Educación para la Integración social de la población afectada por la erupción volcánica en Chaitén. Coordinadora de proyecto Proyección al Medio Externo (PME) "Desplazamiento en cenizas", Centro de Investigación en Vulnerabilidades y Desastres Socionaturales, (CIVDES), Núcleo Científico Milenio NS100022, anita.fuentes.pino@gmail.com 


\section{RETURNING TO CHAITÉN: PARTICIPATORY DIAGNOSTIC OF AN EDUCATIONAL COMMUNITY DISPLACED BY A SOCIONATURAL DISASTER}

\section{ABSTRACT}

Chile is a country characterized by socionatural disasters that regularly affect its territory, including earthquakes, tsunamis, floods, forest fires and volcanic eruptions. In 2008 the eruption of the Chaitén volcano forced the evacuation of more than 1,500 families, who undertook a long process of forced displacement, and in many cases a subsequent return. This complex process also included institutions such as schools and their educational communities.

This article describes a participatory diagnosis made in the context of a process of intervention/ research by an intervention team from the University of Chile, with members of the Juan José Latorre educational community, first school to open in Chaitén after the return in 2010. The results of this participatory diagnosis gave us an overview of the process experienced by this community that allows us to present the educational context before the eruption, during the displacement and subsequent return. Making visible the importance of the school as a focus of attraction for the return of many families. We discuss the methodological approach strategies used and the issues arising from this research process, emphasizing the importance of investigating the effects on mental health resulting from these events in the educational community, and their impact on interpersonal relationships, as well as the new educational demands that arise in this context of the return. Finally, we analyze the pertinence of this methodology of intervention/research in the psychosocial reconstruction processes after socionatural disasters.

KEY WORDS: Displacement, socionatural disasters, volcanic eruption, Chaitén, Chile.

\section{CONTEXTO: EL PROCESO DE DESPLAZAMIENTO Y RETORNO VIVIDO POR LA COMUNIDAD CHAITENINA}

La repentina erupción del volcán Chaitén, iniciada el 2 de mayo del 2008, obligó a las autoridades a declarar Estado de Catástrofe iniciándose así un proceso de evacuación de la totalidad de sus habitantes tanto en Chaitén como es zonas aledañas, trasladándose en diversos buques de la armada y barcos de particulares a más de 6.000 habitantes en menos de 72 horas (Ministerio del Interior, 2008). A los pocos días, se produce el desborde del Río Blanco cambiando su curso de manera definitiva, dividiendo a la ciudad en dos zonas (que actualmente se conocen como sector norte y sector sur) y destruyendo aproximadamente 200 casas y anegando el 92\% del territorio (Maldonado, 2008).

En el presente artículo se presentan los resultados del proyecto "Educación para la integración social de la población afectada por la erupción volcánica en Chaitén", que corresponde a un pro-

1 CIVDES Es un centro de investigación aplicada en ciencias sociales, de carácter inter-disciplinario, cuyo principal objetivo es el estudio del riesgo y de los factores de yecto de extensión universitaria desarrollado entre marzo de 2012 y julio de 2013. La iniciativa forma parte de un fondo concursable de la Vicerrectoría de Extensión de la Universidad de Chile a través de su Fondo Valentín Letelier 2012 y forma parte también del trabajo del Centro de Investigación en Vulnerabilidades y Desastres Socionaturales (CIVDES) ${ }^{1}$.

\section{Efectos psicológicos y psicosociales de los desastres socionaturales}

En relación a los efectos psicosociaes a nivel individual, se advierte que en este tipo de desastres las personas se ven enfrentadas a un suceso que sobrepasa su capacidad habitual de responder efectivamente frente a un evento estresante (Arriagada \& Valdebenito, 2011; OPS, 2006). Arriagada y Valdebenito (2011), describen la presencia de aspectos sintomatológicos que podrían tener lugar en las personas afectadas, tales como: a) alteraciones cognitivas (dificultad

vulnerabilidad (social, económica, cultural) en comunidades afectadas por desastres socionaturales en contextos tanto urbanos como rurales de nuestro país. 
para pensar organizadamente, re-experimentación del evento); b) alteraciones de índole emocional (labilidad emocional, aplanamiento afectivo, intensificación de emociones como rabia, miedo o ansiedad generalizada); c) alteraciones conductuales (impulsividad o paralización, aumento del consumo de alcohol), y d) alteraciones físicas y fisiológicas (dolores corporales, trastornos de sueño o del apetito). Además, generalmente se experimentarian alteraciones en el ámbito relacional-familiar, laboral, comunitario y/o social, expresadas en cambios en la forma habitual de relacionarse, emergencia de conflictos latentes y culpabilización mutua, entre otros.

\section{Efectos psicosociales a nivel comunitario}

Según la Organización Panamericana de la Salud (OPS, 2006), se entiende por impacto psicosocial de los desastres, a los efectos que éstos provoquen en el ámbito psicológico individual, familiar y social de las poblaciones afectadas. Desde el punto de vista de la salud mental, las catástrofes implican que lo psicosocial se ve alterado sobrepasando la capacidad de afrontamiento de la población afectada (OPS, 2006), no siendo posible responder a este fenómeno con los mecanismos habituales de resolución de problemas (Arriagada \& Valdebenito, 2011). Asimismo, el efecto de desestructuración y ruptura del tejido social que este tipo de evento provocan sobre la población afectada, les impediría continuar su funcionamiento cotidiano con normalidad (Arriagada \& Valdebenito, 2011; Beristain, 2000).

En la configuración de la cualidad de los impactos psicosociales que produce un evento catastrófico, las principales investigaciones concluyen que intervendrían tres factores primordiales: a) la naturaleza misma del evento (socionatural o antrópico); b) las características de personalidad de los afectados y vulnerabilidades previas, y c) las circunstancias de ocurrencia del evento (Arriagada \& Valdebenito, 2011; OPS, 2006; Páez et al. 2001).

Con respecto a los efectos producidos por el desplazamientos, diversos autores se refieren a los impactos psicosociales ocasionados por el proceso migratorio de la población desplazada en la erupción volcánica de Chaitén (Espinoza, 2014; Lillo,
2013; Marchant, 2010; Ramírez, 2014). Entre estos efectos destacan: a) las dificultades para asentarse y adaptarse a nuevas comunidades, afectando de igual forma a diversos grupos etáreos (Espinoza, 2014; Lillo, 2013; Marchant, 2010; Ramírez, 2014); b) la destrucción de las redes de apoyo y estilo de vida previo (Marchant, 2010), y c) la atribución de este proceso como causa de enfermedades físicas y psiquiátricas en los afectados e incluso la muerte (Espinoza, 2014; Lillo, 2013).

\section{El contexto actual: la escuela desplazada, la escuela retornada.}

Para comprender la situación actual de la escuela en Chaitén, es importante hacer el recorrido del desplazamiento y retorno que también se vive como institución. Previo a la erupción, existían tres establecimientos educacionales en Chaitén: la escuela básica Almirante Juan José Latorre (en adelante J.J. Latorre), la escuela básica Luz del Corcovado y el Liceo Italia, estando los últimos dos ubicados en el ahora "sector sur" de la ciudad.

Con el desplazamiento, el gobierno reubicó a la gran mayoría de los trabajadores públicos en otras localidades, sin embargo, los profesores no pudieron ser reubicados debido a su condición de trabajadores municipales. En esta condición, siguieron recibiendo sus remuneraciones durante el año 2008, pero con la imposibilidad de trabajar en otras localidades. Sin embargo, cuando se declara la inhabitabilidad de la ciudad de Chaitén, se inició un proceso de finiquito de esta planta docente. El mismo año 2008, parte de estos trabajadores se trasladaron a la localidad de Palena, en donde el Liceo Italia fue anexado a la escuela Roberto White, es decir, el Liceo Italia, administrativamente perteneciente a la Municipalidad de Chaitén, retoma sus funciones con su propia planta de profesores de enseñanza media, en las dependencias de la escuela básica de Palena.

Con el proceso de retorno y a través de una "extensión" ministerial para poder incluir enseñanza media, se produce la reapertura de la Escuela J. J. Latorre a partir del año 2010. En la conformación de esta nueva escuela se produce una fusión de los tres establecimientos educacionales que existían previo a la erupción, en ella confluyen profesores, asistentes de la educación, estudiantes y apodera- 
dos incorporándose también nuevos alumnos y sus familias quienes han llegado a la ciudad sin haber vivido el proceso de desplazamiento y retorno.

\section{EDUCACIÓN EN SITUACIONES DE DESASTRES}

Desde que la Organización de las Naciones Unidas (ONU) declaró la década de los '90 como "La Década Internacional para la Reducción de los Desastres Naturales" (Muñoz, 2010), se han gestado diversas iniciativas internacionales y latinoamericanas que consideran que la gestión del riesgo y la prevención de desastres es una materia central para el desarrollo (Campos, 2000). La atención en la educación cobra sentido pues ésta suele ser interrumpida o postergada en situaciones de crisis, en especial cuando las escuelas se transforman el albergues por mucho tiempo. Esto se ha atribuido a la falta de relación entre las estructuras sociales, culturales y económicas y las intervenciones pedagógicas realizadas en tiempos de conflicto y desastres naturales (Muñoz, 2010).

Asimismo, se ha considerado que, a través de la educación, es posible ir más allá de la gestión del riesgo y avanzar en la creación de una cultura de prevención (Campos, 2000) y que, mediante los procesos de enseñanza-aprendizaje, la escuela permite a los estudiantes ajustarse a las pérdidas y externalizar emociones, además de ofrecer espacios seguros para aprender, así como la capacidad para identificar y proveer apoyo a las personas afectadas, incluyendo también a las familias (Dettmer, 2002). Además, se estima que este contexto atenúa los impactos psicológicos dando un sentido de normalidad y estabilidad durante una época de crisis (Muñoz, 2010; Prinsteinet al. 1996; Vélez, 2011).

Frente a los desastres, las escuelas son demandadas de diversas formas y desde distintos organismos. La principal preocupación de los Estados es reanudar rápidamente las clases ya sea por proteger el derecho a la educación, por mantener los estándares productivos, o por reanudar las rutinas locales, proporcionando de esta forma un sentido de normalidad (Muñoz 2010; Vélez, 2011). Las principales estrategias utilizadas en Chile para este propósito son las fusiones de escuelas y las reubicaciones en otros centros en caso de desplazados. Es así como luego del terremoto y tsunami del 2010, la meta fue que en menos de 50 días de iniciado el año escolar, todo los alumnos se reincorporaran a las escuelas con normalidad (Lavín, 2010). Para esto, la principal acción fue la fusión de escuelas, es decir, que dos o más establecimientos funcionen en el mismo local (EPE, 2010). De manera similar, en el caso de Chaitén, luego de la evacuación masiva tras la erupción, la primera meta era reubicar a los estudiantes y docentes en otros centros educativos de las ciudades a las cuales se habían trasladado. Posteriormente se fusionan las tres escuelas que existían antes del desplazamiento conformando la Escuela J. J. Latorre.

\section{Impactos de los desastres}

en la escuela y sus actores

El contexto de desastres, por tanto, puede influir en el enfoque pedagógico de la actividad escolar en particular y en los procesos de reconstrucción social en general (Muñoz, 2010). Es importante considerar que cuando estos cambios ocurren de manera forzada o repentina, puede haber consecuencias en la comunidad educativa y en la actividad innovadora de padres y profesores (Romero, 2000). Interesa de sobremanera este tema, pues se ha considerado que las reubicaciones y desplazamientos de personas luego de desastres es la situación que más consecuencias negativas tiene para el tejido social, pues, los procesos migratorios, aún cuando se realicen en condiciones favorables, implican para los afectados el experimentar una crisis que puede tener efectos psicosociales profundos y de larga duración (Bar de Jones, 2001; Briones, 2010; Marchant, 2010; ONEMI, 2006). Más aún, cuando éstas son catalogadas como "migraciones forzosas" (Sarrible, 2009), es decir, son repentinas, obligatorias y se mantienen por períodos prolongados de tiempo. Este tipo de desplazamiento tiene repercusiones en la salud física, mental y social de los afectados, en donde el principal problema es el desarraigo, ya sea geográfico, cultural o afectivo (Rastrepo, 1999 citado en Rodríguez, 2007), y en donde se ven afectadas las tres dimensiones del sujeto: individual, grupal o familiar y cultural (Bar de Jones, 2001).

En estos contextos de crisis, y a pesar del lugar central que se le otorga a las escuelas, éstas 
no disponen de sistemas de apoyo para estudiantes en situación de desplazamiento, menos aún para los docentes quienes deben responder a las demandas de apoyo de la comunidad (Cortina, 2012; Markham, 2012). Se ha estimado que la importancia de los profesores en la vida de los niños se incrementa en situaciones de desastres, principalmente por que se ven enfrentados a la desintegración familiar, historiales de estudios interrumpidos, repitencia, retraso en la adquisición de contenidos, además del impacto psicosocial provocado por el proceso de desplazamiento forzado en los estudiantes y sus familias, en donde los padres presentan, en general, una capacidad limitada para apoyar a sus hijos en el proceso educativo (Markham, 2012; Winthrop \& Kirk, 2005). Como consecuencia de lo anterior, la mayoría de los maestros necesitan apoyo para trabajar con niños que han experimentado directa o indirectamente eventos traumáticos, tales como el desplazamiento, la desintegración familiar o el desarraigo (Winthrop \& Kirk, 2005), dado que en estos contextos los profesores carecen de la formación necesaria para atender las diversas necesidades que presentan los estudiantes desplazados (Markham, 2012).

En suma, cuando se habla de educación en situaciones de emergencia, el acento está puesto en la posibilidad de determinar los tipos de atención que entrega la escuela, así como de desarrollar planes de educación en crisis, con medidas concretas para la educación antes, durante y después del desastre (Muñoz, 2010). Sin embargo, considerando la escasa producción de conocimientos en cuanto a los impactos de los desastres socionaturales en la institución escuela, para el presente proyecto de intervención/investigación, parece interesante indagar en el marco de los procesos que atraviesa la escuela al asumir las exigencias descritas, y en particular en un complejo proceso que involucra desplazamiento, asentamiento temporal y retorno.

\section{METODOLOGÍA DE ACCIÓN COMUNITARIA}

Para el proyecto, se trabajó en la elaboración del proceso vivido por los miembros de la comunidad educativa de la escuela J.J. Latorre. El proyecto abordó la problemática de desintegración social derivada del desplazamiento forzado de toda la localidad, con especial atención en la promoción de herramientas que permitieron consolidar una comunidad más resistente física, psicológica y socialmente a las amenazas socionaturales, y promover saberes sobre la relación con el territorio, integrando experiencias y conocimientos locales como un recurso de cohesión social para la resistencia a nuevas amenazas. Con este propósito se diseñó e implementó un programa educativo-participativo que incluyó tres líneas estratégicas de acción: a) la elaboración de la experiencia traumática; b) la consolidación de aprendizajes en torno al enfrentamiento de riesgos socionaturales, y c) la promoción de procesos de resiliencia comunitaria, basados en la integración social de las comunidades desplazadas.

\section{Algunos antecedentes}

El proceso de diagnóstico participativo es el resultado de un proceso de diálogo entre el equipo de intervención/investigación y los representantes de la comunidad educativa de la escuela. La relación de trabajo comienza cuando miembros del equipo contactan al director para comenzar el proceso de postulación a los Fondos Concursables Valentín Letelier de la Vicerrectoría de Extensión de la Universidad de Chile. Inicialmente el director se demostró interesado y manifestó su preocupación por el comportamiento de algunos alumnos retornados a Chaitén y nos planteó la necesidad de que estos alumnos pudieran ser evaluados. También propuso que posteriormente pudiéramos proporcionarles ciertos lineamientos de cómo los profesores pudieran trabajar con ellos. Estas y otras demandas forman parte de un proceso conjunto de identificación de necesidades que sirvieron de base para el diseño de las intervenciones individuales de atención psicológica especializada y de contención psicosocial que conforman el diagnóstico participativo. Asimismo, estos primeros acercamientos son parte de un proceso de construcción de confianzas que se fueron fortaleciendo durante los dos años que el equipo trabajó en Chaitén.

\section{Estrategia metodológica}

La estrategia metodológica utilizada fue el diagnóstico participativo que permitió poder comprender la experiencia del desplazamiento 
y retorno de los diferentes actores educativos y el impacto en la salud mental de éstos, así como también sus necesidades y demandas a nivel laboral. El diagnóstico participativo se plantea como una herramienta con un doble propósito para la intervención en este contexto: por un lado, permite ingresar a la comunidad, conectarse con sus necesidades y establecer un vínculo de confianza, y con ello generar información para planificar las intervenciones (Mori, 2008). Por otra parte, permite que la actividad en sí se transforme en una intervención de tipo terapéutica, al trabajar con elementos significativos, legitimando así la experiencia y conteniendo emocionalmente a los participantes a través de un trabajo creativo a partir de técnicas derivadas de las terapias del arte.

\section{Validación de la experiencia y saberes}

Un elemento central de esta metodología de tipo participativa es la validación de la experiencia de quienes pertenecen a la comunidad, dado que son los conocedores de su propia realidad y de los problemas que los afectan. Por lo tanto, son ellos los que tienen la capacidad para reconocer sus problemas y necesidades ordenando o priorizando áreas de intervención de acuerdo a criterios comunes para motivar a la comunidad en la búsqueda de soluciones compartidas que lleven a la solución de sus problemas (Mori, 2008; Valdés, 2006).

\section{EL DIAGNÓSTICO PARTICIPATIVO COMO PROCESO EDUCATIVO}

El diagnóstico entendido como una experiencia educativa en sí misma, permite compartir experiencias, intercambiar conocimientos o aprender a utilizar técnicas para recoger información. Como en todo proceso educativo participativo, el diagnóstico parte necesariamente de los conocimientos y experiencias de los participantes. En este sentido, la vivencia diaria, proporciona a la gente una base de conocimientos de su propia realidad. Es sobre esta base que se inicia el proceso de reflexión y la búsqueda de profundización en los niveles de información. Una condición básica para una efectiva participación en el proceso diagnóstico es asegurar que todos los participantes tengan la suficiente información acerca de los objetivos, la metodología y los mecanismos de participación de cada uno de los actores involucrados (Mori, 2008; Valdés, 2006).

\section{Terapias del arte}

Un complemento importante a esta metodología lo constituyen las técnicas derivadas de las terapias del arte. De acuerdo con Bourriaud (2006), el arte tiene el potencial de gatillar no sólo procesos de creación colectiva, sino también de reflexividad social, dado que las actividades artísticas son una expresión natural de los seres humanos para dar a conocer sus necesidades, experiencias y emociones, facilitando de esa forma comunicación intra e interpersonal. En estas prácticas artísticas se enfatiza la intersubjetividad, de un estar junto al otro. La obra se construye de manera colectiva, entre creador y espectador (Bourriaud, 2006). Es por ello, que las terapias de arte, nos ayudan a comprender lo que observamos; constituyen medios sensibles que permiten una perspectiva de aproximación desde la propia realidad sociocultural de las personas. A lo anterior se suma, el potencial relacional del arte y la creatividad en intervenciones psicosociales. En este contexto, el interventor/investigador usa creativamente los medios artísticos como vehículo para una comunicación no verbal y/o simbólica, dentro de un contexto de apoyo y contención emocional (Karkou \& Sanderson, 2006).

\section{Participantes}

Para efectos de este diagnóstico se consideró a toda la comunidad educativa como participantes, aproximadamente unas 225 personas. Se trabajó por separado con cada uno de los estamentos educativos, con cada curso de alumnos de la escuela, desde primero básico a cuarto medio, docentes, apoderados y también funcionarios y asistentes de la educación. La primera intervención desarrollada parte de la siguiente consigna ¿cómo estoy hoy en Chaitén?, la cual fue abordada utilizando diferentes estrategias derivadas de las terapias del arte según el grupo etáreo; desde la utilización de títeres y dibujos con el primer ciclo básico; historias con educación media, y collages con el segundo ciclo, apoderados, funcionarios y docentes. En la utiliza- 
ción de estas técnicas para trabajar en los talleres, se considera tanto los productos finales creados por los participantes, así como el proceso creativo y la narración asociada, lo que permite una comprensión mucho más profunda de la experiencia de las personas. En total se realizaron 4 talleres con docentes donde participaron en promedio 20 profesores; 11 talleres con alumnos de enseñanza básica y media con un total de 178 alumnos; 3 talleres con padres y apoderados con un promedio de 15 participantes y 3 talleres con asistentes de la educación donde participaron 12 personas en promedio. El análisis del material se realizó a partir de la transcripción de los talleres, de las observaciones registradas en cada intervención y como parte de un proceso de discusión y reflexión del equipo, que se realizaba en forma inmediatamente posterior a las intervenciones. Esta información era devuelta a los participantes en las siguientes intervenciones, generando instancias de reflexión que llevaban a la identificación de sus propios recursos, así como de nuevas necesidades.

En relación a la solicitud inicial por parte del director de realizar un diagnóstico clínico de algunos estudiantes, se trabajó en duplas primero realizando la evaluación clínica de estos estudiantes en forma individual y posteriormente con sus padres a través de entrevistas clínicas. La información de estos diagnósticos fue analizada por el equipo clínico generando recomendaciones para cada uno de los casos, las que fueron enviadas al director de la escuela. Se realizó también un taller con los padres o madres de los niños y adolescentes, así como una intervención específica con los niños, a fin de dar respuesta a las necesidades psicológicas evidenciadas a partir de las entrevistas.

\section{RESULTADOS Y DISCUSIÓN}

Los resultados de este diagnóstico participativo que aborda tres líneas de acción vinculadas a iniciar un proceso de elaboración de la experiencia traumática; el fortalecimiento de aprendizajes relacionados al enfrentamiento de riesgos socionaturales y el desarrollo de procesos de resiliencia comunitaria. Al respecto, los hallazgos se pueden dividir en dos líneas temáticas: a) la experiencia de los miembros de la comunidad educativa y los efectos en la salud mental de este proceso de desplaza- miento, asentamiento temporal y retorno y cómo éstas se relacionan con los procesos institucionales, y b) la pertinencia del diagnóstico participativo como estrategia metodológica con poblaciones afectadas por desastres socionaturales.

El diagnóstico participativo realizado, permite levantar una panorámica general de la situación actual de la escuela en Chaitén. Frente a esto, nos parece importante caracterizar el retorno como un proceso en desarrollo pues, aunque se inicia oficialmente a partir del año 2010, actualmente la población continúa regresando de forma continua. Uno de los primeros hallazgos a partir de las intervenciones da cuenta de la necesidad de los miembros de esta escuela de relatar las experiencias vividas. En este sentido, el proceso de evacuación surge como el evento traumático más importante en las vidas de estas personas. La evacuación ocurrida en horas de la noche en un contexto que identifican como caótico, es vivida con mucho miedo, ansiedad y confusión, ya que se desconoce hacia donde serán trasladados y cuándo podrán regresar. Esta experiencia se identifica como el inicio de los problemas de salud mental para muchas personas, lo que se traduce en estados ansiosos y depresiones que se mantienen por mucho tiempo. De igual forma, la falta de información y comunicación por parte de las autoridades se transforma en un sentimiento de molestia el que se va agudizando conforme pasan los meses y se exacerba cuando Chaitén es declarado inhabitable y comienzan los estudios para el reasentamiento de la población en la localidad de Santa Bárbara. Estas noticias son vividas con mucho desconsuelo e incertidumbre ya que, entre otras cosas, significa el desarraigo permanente de su territorio y por tanto una profunda pérdida de su identidad territorial. Estos hallazgos son compartidos por Larenas (2012) quien sostiene que la comunidad chaitenina actual aparece como muy cohesionada con una fuerte identidad territorial, en donde parece haber un espíritu común que hace referencia a los primeros colonos que poblaron la zona, que impregna una convicción de que el pueblo, y sus vidas, es resultado de su propio esfuerzo, que es un territorio indisociable de su identidad, y que por lo tanto, su vida sólo ahí tiene sentido.

La incertidumbre laboral y la inserción a otros establecimientos educacionales es otro tema que afectó a los miembros de esta comunidad 
de desplazados y que los lleva a tomar diversas decisiones en relación a permanecer en sus lugares de asentamiento temporal o regresar a Chaitén. En general, la experiencia del desplazamiento es vivida con mucha tristeza y confusión producto del desarraigo y la ruptura del tejido social, lo que los lleva a sentirse como extranjeros en su propia tierra. En el caso de los docentes y técnicos de la educación que emigraron a Palena, la experiencia laboral, si bien es vivida con entusiasmo, los confronta con otras prácticas pedagógicas y en general una cultura organizacional que les es diferente, dificultando los procesos de reinserción laboral y acrecentando los problemas de salud y salud mental. De igual forma, la experiencia de las familias desplazadas, muchas de ellas separadas, está también marcada por episodios de discriminación y matonaje al interior de las escuelas, lo que los lleva a una experiencia de mucha soledad y aislamiento. Por lo tanto, la reapertura de la escuela J.J. Latorre representa una posibilidad concreta de volver al territorio y a un contexto conocido y querido en donde la Escuela se transforma en un foco que atrae a muchas de las familias retornadas.

Estos resultados son confirmados por Lillo (2013), quien identifica que durante el proceso de desplazamiento, es frecuente una vivencia de lo escolar caracterizada por sufrimiento, segregación, discriminación y matonaje, tanto por otros estudiantes, como a un nivel institucional, configurando una serie de desencuentros culturales y un historial de itinerancia escolar en los afectados. Sin embargo, en el proceso de retorno, se presenta la escuela de Chaitén como una brújula de la migración, considerándose la reapertura de la escuela como condición, sin la cual el proceso de retorno no se hubiese producido de manera masiva. Constituyéndose al mismo tiempo como un elemento que permite la unificación familiar de aquellas familias que se habían fragmentado durante el desplazamiento (Lillo, 2013). Por otra parte, el proceso de retorno no ha sido fácil, ya que ha implicado el retornar a una ciudad que no es la misma no sólo en su contexto físico, sino que también la ausencia de muchos amigos, vecinos y familiares aún produce un impacto a nivel emocional, generando un segundo duelo que reabre procesos no resueltos que interfieren en sus relaciones laborales y sociales.

Respecto a la escuela como institución retor- nada nuestro análisis nos revela que se encuentra atravesada por un proceso de cambio constante que se manifiesta tanto a nivel institucional como a nivel interpersonal. A nivel estructural, este cambio está dado por la fusión de las tres escuelas que existían previamente en Chaitén, lo que ha implicado una reestructuración de funciones y prácticas laborales. Lo anterior ha significado para la comunidad educativa adaptarse a una escuela que ya no es la misma donde trabajaban antes de la erupción. A nivel personal, cada persona y familia trae consigo experiencias individuales y colectivas del desplazamiento, elementos que ciertamente influyen en la articulación de las relaciones interpersonales de los diferentes actores de este centro educativo.

Un elemento transversal que surge en distintas etapas del diagnóstico es la necesidad que demuestran los miembros de esta comunidad de contar sus experiencias, de ser escuchados y validados en su dolor. Si bien los participantes recuerdan haber tenido intervenciones psicosociales durante la etapa de la emergencia, manifiestan que esta ayuda fue esporádica y corta, pero que no habían tenido la oportunidad de elaborar las experiencias vividas hasta estas intervenciones. En este sentido, manifiestan sentirse abandonados por el Estado en este proceso de reconstrucción, y en particular, después del terremoto y tsunami del 2010, ya que la atención por parte de las autoridades del Estado se trasladó a otras partes del país.

Esta falta de apoyo y de espacios de contención y reflexión tiene importantes repercusiones en el ámbito laboral ya que como identificamos, muchos factores relacionados con la falta de comunicación entre los diferentes estamentos de la escuela provenía del hecho que pertenecían a las tres escuelas y liceos de Chaitén, pero no se conocían personalmente ni tampoco sus experiencias de desplazamiento, lo que hacia difícil el desarrollo de confianzas mutuas. Lo anterior esta profundamente vinculado con lo que plantea Ball (1989) en relación a la fusión de escuelas en contextos de post-desastre como estrategia, pues es una situación que genera conflictos de poder entre los distintos actores, así como también afecta considerablemente el clima escolar y modifica las prácticas cotidianas. De igual forma, se pudo identificar que estos procesos se implementan, generalmente, sin un trabajo de coordinación y apoyo a los trabaja- 
dores de la educación, ni considerando el impacto que esto pueda tener en las comunidades educativas, muchas de ellas arraigadas e identificadas con sus escuelas de origen como lo ha investigado el Equipo de Psicología y Educación de la Universidad de Chile (EPE, 2010).

Por otra parte, la experiencia concreta de los profesores surge como un ámbito de indagación de suma importancia. Las experiencias de estos profesionales refleja la diversidad de roles que como maestros deben asumir en contextos rurales y de aislamiento como el caso de Chaitén. En estos contextos, los profesores pasan a formar parte integral de la comunidad, estableciendo fuertes vínculos personales con sus alumnos, padres y apoderados. Por lo tanto, frente a la emergencia asumieron fuertemente el rol de proporcionar contención emocional a la comunidad durante todo el proceso de desplazamiento, asentamiento y retorno. Sin embargo, a nivel personal manifiestan que no recibieron la ayuda y contención emocional que han necesitado para seguir realizando sus funciones en la etapa de retorno. Lo anterior abre un interesante debate en relación a lo planteado por la literatura sobre el rol de la escuela en las etapas de mitigación, ajuste y recuperación en situaciones de desastres (Dettmer, 2002) al ser pensados como espacios de encuentro y formación entre estudiantes, docentes y la comunidad (Montero et al. 1998; Muñoz, 2010), ya que como hemos señalado estos profesionales no se encuentran necesariamente en condiciones de asumir estos desafíos sin un proceso de acompañamiento psicosocial que los ayude a asumir estas responsabilidades.

Esta revelación abre una interesante línea investigativa respecto a los roles de estos profesionales en contextos de emergencia, así como la necesidad de proporcionar ayuda psicológica y psicosocial a quienes trabajan conteniendo a la comunidad en primera línea. Sin lugar a dudas la información recabada a partir de estas intervenciones demuestra la importancia de proporcionar intervenciones psicosociales a comunidades afectadas por desastres socionaturales, dado que como lo demuestra nuestro trabajo en Chaitén, los miembros de esta comunidad educativa estaban aún fuertemente afectados por la experiencia vivida a cuatro años de la tragedia. Estos hallazgos coinciden con Pérez-Sales (1998), quien plantea que las investigaciones de los últimos años apuntan, cada vez con más fuerza, a la importancia de aquellas condiciones $e$ intervenciones que tienen lugar post-desastre, las cuales representarían otro factor de incidencia en la configuración del impacto psicosocial de los desastres socionaturales.

\section{Diagnóstico participativo como estrategia metodológica}

Los resultados indican que el diagnóstico participativo como estrategia metodológica resulta ser pertinente en contextos investigativos con comunidades que han sido afectadas por desastres socionaturales. Al respecto este tipo de intervenciones cumple tres propósitos:

a) Permite entrar a la comunidad a través del reconocimiento de las experiencias de sus miembros lo que permite generar lazos de confianza. Se produce entonces, una construcción de demandas en forma conjunta. En este espacio se hace necesario que los investigadores puedan negociar y clarificar las expectativas, teniendo la precaución de no hacer falsas promesas, las que no puedan ser cumplidas posteriormente, perjudicando de esta forma los lazos de confianza y el trabajo en general.

b) El diagnóstico participativo es una estrategia de producción de datos que permite levantar información en cada actividad, profundizando así el material obtenido a partir del contexto participativo en el que es generado y dados los procesos de retroalimentación y evaluación realizados con los participantes. Estas estrategias implican una revisión permanente de los objetivos y de la pertinencia de las intervenciones que se planean, teniendo presente que la demanda está siempre en construcción y puede ser reformulada. Nuestra experiencia demuestra que los equipos de intervención deben ser capaces de identificar y validar las experiencias de los sujetos y desde ahí realizar una intervención con sentido para la comunidad, ya que son estos actores quienes tienen la mayor capacidad para reconocer, ordenar o priorizar y solucionar sus problemas. 
c)

\begin{abstract}
Consideramos también que este tipo de estrategia se transforma en un espacio terapéutico para los participantes. El trabajo desde las terapias del arte permitió generar un dispositivo de escucha y contención para los participantes, los que a través de diversas técnicas fueron capaces de comenzar un proceso de elaboración de sus experiencias a partir de una reflexión sobre los productos creados. Estas estrategias han probado ser menos intrusivas que otras estrategias de intervención basadas solamente en el relato de experiencias traumáticas.
\end{abstract}

Este proceso de intervención/investigación desarrollado a partir de un diagnóstico participativo permitió iniciar un proceso de elaboración de la experiencia traumática a través de intervenciones grupales basadas en las terapias del arte, lo que contribuyó a una reflexión conjunta en torno a los procesos de afrontamiento de riesgos socionaturales. En este contexto, se identifica la identidad territorial como un elemento clave en la conformación de la resiliencia comunitaria de esta población. Asimismo, se identifica la importancia de continuar investigando el rol de la escuela y su comunidad educativa en contextos de desastres socionaturales y en particular las necesidades psicosociales de sus miembros.

\section{CONCLUSIONES}

Los resultados obtenidos a partir de este diagnóstico participativo reflejan una serie de temáticas que emergen de la participación de los miembros de esta comunidad educativa, sus necesidades y demandas, lo que constituye la base para las intervenciones posteriores. Estas estrategias interventivas permitieron identificar cómo este proceso vivido tras la evacuación ha tenido un efecto importante en la salud mental de los chaiteninos. De esta forma se identifica que el contexto caótico de la evacuación, unido a la falta de información clara por parte de las autoridades a cargo genera altos niveles incertidumbre y de ansiedad en toda la población respecto a su destino, iniciándose así diversos problemas de salud mental. Estos problemas se vieron acentuados por las experiencias vividas en los lugares de asentamiento temporal, dado que muchos de ellos no lograron adaptarse a las costumbres, la cultura y el entorno, aumentando en muchos casos los problemas de salud mental existentes. Por otra parte, el retorno a Chaitén significó reabrir temas no resueltos obligando a muchos a vivir nuevos procesos de duelo. Cabe mencionar la importancia de la elaboración de este tipo de eventos con las comunidades afectadas, y en particular aquellos actores comunitarios, como los profesores y directivos de las escuelas, quienes, en estos contextos, asumen nuevos roles, lo que conlleva una enorme carga emocional para ellos. $\mathrm{Al}$ respecto, cabe enfatizar que este tipo de trabajo psicosocial debería asumirse como un trabajo previo y necesario antes de iniciar cualquier otra iniciativa de prevención de nuevas emergencias y desastres socionaturales.

A nivel metodológico, las estrategias participativas implementadas demostraron ser pertinentes dentro del trabajo realizado con esta comunidad, permitiendo identificar importante información respecto a los procesos vividos, su impacto en la vida y salud mental de las personas, así como también en las dinámicas institucionales y en las prácticas de esta comunidad educativa. Asimismo, se logra establecer que los procesos de reconstrucción comunitarios requieren de metodologías participativas flexibles, que permitan ser modificadas a medida que surgen nuevas necesidades y demandas. La retroalimentación entregada a la comunidad luego de cada intervención permite también establecer un proceso continuo de evaluación y reformulación de nuevos objetivos en forma conjunta. Por último, toda esta información configura un escenario desde donde comenzar a cuestionar las expectativas que se han ido generando en torno al rol de la escuela y su comunidad educativa en contextos de desastres socionaturales, aportando nuevos antecedentes en torno a la pertinencia de establecer protocolos de capacitación y acompañamiento psicosocial de los miembros de estas comunidades, quienes, a menudo, son incluidos en los procesos de reconstrucción por parte del Estado, pero sin el apoyo adecuado que demandan estos procesos.

\section{BIBILIOGRAFÍA}

Arriagada, P., \& Valdebenito, L. (2011). Para reconstruir la vida de los niños y niñas. Guía para apoyar 
intervenciones psicosociales en Emergencias $y$ Desastres. Santiago: Fondo de las Naciones Unidas para la Infancia, UNICEF.

Bar de Jones, G. (2001). Panel Quiebres Vitales: La Migración como Quiebre Vital. En II Congreso Argentino de Psicoanálisis de Familia y Pareja. Teoría y Clínica de los Vínculos.

Beristain, C. (2000). Apoyo psicosocial en catástrofes colectivas: de la prevención a la reconstrucción. Caracas: Asociación Venezolana de Psicología Social.

Bourriaud, N. (2006). Estética relacional. Buenos Aires: Editorial Los sentidos/artes visuales.

Briones, F. (2010). Inundados, reubicados y olvidados: Traslado del riesgo de desastres en Motozintla, Chiapas. Revista de Ingeniería Universidad de Los Andes. Bogota, Colombia, 31, 132-144.

Campos, A. (2000). Educación y prevención de desastres. Fondo de las Naciones Unidas para la Infancia. Facultad Latinoamericana de Ciencias Sociales; Red de Estudios Sociales en Prevención de Desastres en América Latina.

Cortina, E. (2012). Prácticas docentes en contextos con población en situación de desplazamiento en la ciudad de Barranquilla y municipios del departamento del Atlántico-Colombia. IX SEMINÁRIO DA REDE ESTRADO Politicas Educativas Na América Latina: Praxis Docente E Transformação Social, 1-10.

Dettmer, J. (2002). Educación y desastres: reflexiones sobre el caso de México. Revista Latinoamericana de Estudios Educativos, XXXII(2), 43-72.

Espinoza (2014). Aproximaciones a la comprensión de los efectos traumáticos del desplazamiento forzado producto de la erupción del Volcán Chaitén en la población retornada. En Cabrera P. (comp). Construcciones. Clínica de lo Traumático y Figurabilidad, pp 161-171. Santiago, Chile: Colección Praxis Psicológica, Serie Obras de Programas, FACSO / EL BUEN AIRE.

Equipo de Psicología y Educación. (2010). Los desafíos de la escuela en su reconstrucción. Observatorio Chileno de Políticas Educativas. Recuperado de http:// www.opech.cl/inv/analisis/epe_desafios_escuela. reconstruccion.pdf

Karkou, V., \& Sanderson, P. (2006). Defining arts therapies. En V. Karkou and P. Sanderson, (Edis.) Arts Therapies a research based map of the field (pp. 29-48). London: Edit. Elsiever.

Larenas, J. (2012). Algunas reflexiones en torno a las estrategias de intervención del Estado en Chaitén. Centro de Investigación en Vulnerabilidades y Desastres
Socionaturales.

Lavín, J. (2010). A 40 días de cumplirse la meta: En una semana 410 mil alumnos ya se reincorporaron a clases. Ministerio de Educación. Recuperado de http://www. mineduc.cl/index2.php?id_contenido $=10690 \& i d$ seccion $=10$ \&id_portal $=1$

Lillo, M. (2013). Influencia de la reapertura de la Escuela Almirante Juan José Latorre en el proceso de retorno de población desplazada por la erupción volcánica en Chaitén. Tesis de Pregrado, Departamento de Psicología, Facultad de Ciencias Sociales, Universidad de Chile.

Maldonado, J. (2008). Crecida de ríos por material piroclástico de volcán y lluvias deja al 92\% de Chaitén bajo el agua. La Tercera. Recuperado de www.latercera.cl

Markham, L. (2012). Orientación para una juventud reasentada. Revista Migraciones Forzadas, 40.

Marchant, J. P. (2010). Lágrimas de ceniza. Estudio cualitativo sobre la experiencia de desplazamiento de los habitantes de Chaitén, asentados en las ciudades de la Isla de Chiloé y Puerto Montt. Oficina Nacional de Emergencia. Santiago, Chile.

Ministerio del Interior. (2008). Decreto $N^{\circ}$ 588. Señala como afectada por la catástrofe la provincia de Palena. Santiago, Chile.

Montero, A., Rojas, S., \& Montero, D. (1998). El papel del centro educativo en la prevención y mitigación de desastres: Unidad didáctica para docentes. San José, Costa Rica: Centro Regional de Información sobre Desastres para América Latina y el Caribe (CRID).

Mori, M. (2008). Una propuesta metodológica para la intervención comunitaria. Revista Liberabit, 14, 81-90.

Muñoz, V. (2010). El Derecho a la Educación en situaciones de emergencia. Revista Latinoamericana de Inclusión Educativa, 4(2), 59-77.

Oficina Nacional de Emergencia del Ministerio del Interior [ONEMI]. (2006). Intervención Psicosocial en Situaciones de Emergencia y Desastre: Guía para el Primer Apoyo Psicológico. Santiago, Chile.

OPS (2006). Guía práctica de salud mental en situaciones de desastres. En J. Rodriguez, M. Zaccarelli, \& R. Pérez (Eds.). Washington, D.C.: Organización Mundial de la Salud.

Páez, D., Fernández, I., \& Beristain, C. (2001). Catástrofes, traumas y conductas colectivas: Procesos y efectos culturales. En C. San Juan (Ed.), Catástrofes y ayuda en emergencia: Estrategias de evaluación, revención y tratamiento (pp. 85-148). Barcelona: Icaria.

Pérez-Sales, P. (1998). Intervención en catástrofes desde un 
enfoque psicosocial y comunitario. Átopos, 5-18.

Prinstein, M., La Greca, A., Vanberg, E., \& Silverman, W. (1996). Children's coping assistance: How parents, teachers, and friends help children cope after a natural disaster. Journal of Clinical Child Psychology, 25(4), 463-473.

Ramírez, C. (2014). Vulnerabilidades emergentes en poblaciones desplazadas por desastres en dos comunidades: Nueva Cinchona en Costa Rica y Villa El Bosque en Futaleufú, Chile. Tesis para optar al grado de Magíster en Psicología, mención Psicología Comunitaria, Universidad de Chile, Chile.

Rodríguez, E. (2007). El desplazamiento forzado: una reto para transformar la escuela. Estudio de Caso de la Institución Suroriental de la Ciudad de Pereira. Universidad Tecnológica de Pereira.
Romero, F. (2000). Las reformas en educación: Los condicionantes externos. Revista de Ciencias Humanas, 23.

Sarrible, G. (2009). Migraciones forzosas y medioambiente. Reflexiones sobre cuestiones político-sociales más que naturales. Revista de Bioética y Derecho, 17, 29-39.

Valdés, A. (2006). El diagnóstico participativo. Serie de apuntes, asignatura. Modelos de intervención y práctica comunitaria. Escuela de Terapia Ocupacional, Facultad de Medicina, Universidad de Chile.

Vélez, B. (2011). La escuela en tiempos de crisis: Puntos de fuga para re-instaurar la esperanza en contextos postdesastre. Revista Folios, 34, 25-35.

Winthrop, R., \& Kirk, J. (2005). Desarrollo del maestro y bienestar estudiantil. Revista Migraciones Forzadas, 22. 